\title{
Diagnostic Ability of 384-Slice Computed Tomographic Angiography in Prediction of Myocardial Ischemia in Patients with Myocardial Bridging (MB) as Compared to SPECT-MPI Examination
}

\author{
Majid Maleki, ${ }^{1}$ Ali Zahedmehr, ${ }^{2}$ Bahar Galeshi, ${ }^{3}$ Nahid Yaghoobi, ${ }^{4}$ Ahmad Bitarafan-Rajabi, ${ }^{4}$ Maryam \\ Mohammadzadeh, ${ }^{5}$ Ali Abbasian Ardakani, ${ }^{6}$ Ali Pourvaziri, ${ }^{7}$ Dorna Charkheshi, ${ }^{1}$ Ali Kazem Mousavi, ${ }^{3}$ \\ and Ali Mohammadzadeh ${ }^{8, *}$ \\ ${ }^{1}$ Department of Cardiology, Rajaie Cardiovascular Medical and Research Center, Iran University of Medical Sciences, Tehran, Iran \\ ${ }^{2}$ Department of Interventional Cardiology, Rajaie Cardiovascular Medical and Research Center, Iran University of Medical Sciences, Tehran, Iran \\ ${ }^{3}$ Senior Cardiology Resident, Rajaie Cardiovascular Medical and Research Center, Iran University of Medical Sciences, Tehran, Iran \\ ${ }^{4}$ Department of Nuclear Medicine, Rajaie Cardiovascular Medical and Research Center, Iran University of Medical Sciences, Tehran, Iran \\ ${ }^{5}$ Department of Radiology, Amiralam Hospital, Tehran University of Medical Sciences, Tehran, Iran \\ ${ }^{6}$ Department of Medical Physics, Iran University of Medical Sciences, Tehran, Iran \\ ${ }^{7}$ Advanced Diagnostic and Interventional Radiology Research Center, Tehran University of Medical Sciences, Tehran, Iran \\ ${ }^{8}$ Department of Radiology, Rajaie Cardiovascular and Medical Research Center, Iran University of Medical Sciences, Tehran, Iran \\ "Corresponding author: Ali Mohammadzadeh, Department of Radiology, Rajaie Cardiovascular and Medical Research Center, Iran University of Medical Sciences, Tehran, Iran. \\ E-mail: mralimohammadzadeh@yahoo.com
}

Received 2017 July 01; Revised 2017 December 30; Accepted 2018 January 06.

\begin{abstract}
Background: During the past decade, coronary computed tomographic angiography (CCTA) has become the primary non-invasive imaging technique for the assessment of myocardial bridging (MB).

Objectivs: The aim of this study was to evaluate the ability of CCTA to predict myocardial ischemia in patients with MB.

Patients and Methods: A total of 32 MB patients (21 males and 11 females) participated in this study. Eleven MB parameters were measured to assess the ability of CCTA to predict MB patients with ischemia. In order to evaluate ischemia, all the patients underwent single positron emission computed tomography-myocardial perfusion imaging (SPECT-MPI) examination.

Results: Ischemia was observed in 17 patients (53.1\%), while 15 patients (46.9\%) did not show signs of ischemia. Out of the 32 patients, superficial MB was observed in 15 patients while deep MB was identified in 12, and borderline was observed in five patients. All MB examined parameters were found to be significantly different between ischemic and non-ischemic patients, except for the location and tunnel artery diameter in diastole. Moreover, a cut-off value of $0.65 \mathrm{~mm}$ was able to discriminate ischemia with a sensitivity of $100 \%$, specificity of $93 \%$, and yield area under the receiver operating characteristic (ROC) curve (AUC) of 0.996. Also, by considering the depth cut-off value of $1.75 \mathrm{~mm}$, ischemia can be distinguished with sensitivity and specificity of $100 \%$. MB length had a lower discrimination power, with a cut off value of $22.5 \mathrm{~mm}$ yield, $76 \%$ sensitivity, $67 \%$ specificity, and AUC $=0.810$ in the diagnosis of ischemia.
\end{abstract}

Conclusion: CCTA was a reliable modality with high accuracy to depict MB, identify high risk MB, and prevent unnecessary SPECTMPI examination.

Keywords: Computed Tomographic Angiography, Diagnostics, Ischemia, Myocardial Perfusion Imaging, Myocardial Bridging

\section{Background}

In addition to coronary atherosclerosis, miscellaneous risk factors such as hypercoagulability states, coronary artery spasm, congenital anomalies of coronary arteries, endothelial or microvascular dysfunction, severe ventricular hypertrophy, aortic stenosis, and myocardial bridging (MB) may lead to myocardial ischemia (1). Myocardial bridging $(\mathrm{MB})$ is a congenital coronary artery anomaly. It occurs when a segment of an epicardial coronary artery runs into the myocardial wall, beneath the muscle bridge. This may lead to vessel compression during systole and result in myocardial ischemia.

The most frequent location of $\mathrm{MB}$ is in the middle segment of the left anterior descending (LAD) artery $(2,3)$. The depth and length of MB were reported at a range of 1 to 10 $\mathrm{mm}$ and 10 to $30 \mathrm{~mm}$, respectively (4). MB is classified in regard to their depths (superficial: > 1 to $2 \mathrm{~mm}$ vs. deep: $>$ 
$2 \mathrm{~mm})(5)$.

MB was first described by Portman during angiographic imaging in 1960 (6). Since then, coronary arteries have been evaluated by a wide range of newer diagnostic measures such as intracoronary Doppler sonography, magnetic resonance imaging, and coronary computed tomographic angiography (CCTA) (7-11). CCTA has become the primary non-invasive imaging technique for the assessment of MB due to its capabilities such as multi-planar reconstruction, high versatility as well as high accuracy and reliability $(12,13)$.

As previously mentioned, MB could lead to myocardial ischemia. The occurrence of myocardial ischemia correlates with the length and depth of the bridging vessel, since ischemia is more frequent in long and deep MBs (14). Single photon emission computed tomographic -myocardial perfusion imaging (SPECT-MPI) is a clinical standard imaging technique for myocardial perfusion, but it suffers from poor spatial resolution and soft tissue attenuation artifacts. Also, it has a risk of ionizing radiation inherently $(15,16)$.

\section{Objectives}

The aim of this study was to evaluate the potential of CCTA imaging to predict ischemia in patients with MB in comparison with SPECT-MPI imaging.

\section{Patients and Methods}

\subsection{Patients and Image Acquisition}

Patients considered for this study were MB-proven by CCTA at Rajaie cardiovascular Medical and research center between March 2015 and March 2016. This study was conducted with an approval from the ethics committee of the Iran University of Medical Sciences. The inclusion criteria of computed tomography angiography (CTA) and SPECTMPI are described as follows: patients with suspected coronary artery disease who were referred from sub-specialized cardiologists for CCTA and patients diagnosed with MB were selected based on referral to a subspecialized cardiologist to evaluate MB induce ischemia using SPECT-MPI. Patients with active asthma, impaired renal function, drug use which has an effect on the autonomic system, smoking habit, and heart disorders such as coronary artery disease, myocardial hypertrophy, valvular heart disease, and dilated cardiomyopathy, were excluded from this study. Informed consent was obtained from the patients and they underwent stress/rest for SPECT-MPI examination to evaluate ischemic degree.
In order to obtain optimal image quality, the heart rate (HR) of patients was measured before CCTA examination and a heart rate of more than $70 \mathrm{bpm}$ was corrected using 50 to $100 \mathrm{mg}$ metoprolol (Alborz Darou, Iran), which was administered orally. Sublingual nitroglycerin (G.Pohl-Boskamp GmbH and Co, Germany) was also administered for vasodilatation of coronary arteries in order to acquire superior images. All patients were evaluated by Dual-source 384 slice-CT with electrocardiogram (ECG) gated. Contrast administration was executed with a bolus of 30 to $50 \mathrm{cc}$ contrast agent (omnipaque $350 \mathrm{mg} / \mathrm{mL}$ ) and a subsequent flow rate of 4.8 to $5.2 \mathrm{~mL} / \mathrm{s}$ in keeping patient's weight and glomerular filtration rate (GFR) and using 30 to $40 \mathrm{~mL} / \mathrm{s}$ of post contrast saline chaser.

Myocardial perfusion study was carried out in a 2day study protocol. On the first day, a rest study was performed, followed by a stress study (exercise or druginduced) on the second day. In both rest and stress studies, $740 \mathrm{MBq}$ of Technetium-99m-methoxyisobutylisonitrile (99mTc-MIBI) was injected intravenously and ECG-gated SPECT was started $45 \mathrm{~min}$ after injection of 99mTc-MIBI.

CCTA images were acquired from a Dual-source 384 multi-slice CT (Somatom Force, SIEMENS Healthcare, Erlangen, Germany), with prospective ECG-gated. All parameters in CCTA examination are as follows: Collimation width $=384 \times 0.6 \mathrm{~mm}$, tube Voltage $=140 \mathrm{kV}$, tube current $=80$ effective mAs tube effective current $330 \mathrm{~ms}, 500$ to 1000 $\mathrm{MA}$, temporal resolution $=66 \mathrm{~ms}$, and slice thickness $=0.6$ $\mathrm{mm}$. All reconstructions were done between 30\% and 70\% of R-R cycle being the best interval for a systole and diastole phenomenon. In one breath-hold during inspiration, scanning was performed from the tracheal carina to the diaphragm. All CCTA images were reconstructed using an iterative technique in short axis, sagittal, coronal and three dimensional (3D) views.

Data acquisition was done using a dual-head SPECT camera system (symbia T2, SIEMENS Healthcare Erlangen, Germany), consisting of a low-energy, high-resolution, parallel-hole collimator; a 20\% symmetric window at 140 keV photopeak; a $64 \times 64$ matrix; an elliptic orbit with step and shoot acquisition at 64 views over 180 degrees rotation. All SPECT-MPIs were reconstructed using filter back projection technique using a Butterworth filter with a cutoff of 0.45 and order of 5 .

\subsection{Image Interpretation}

All CTA images were viewed, characterized, and analyzed on syngo.via software package (Siemens Healthcare, Erlangen, Germany). CTA images were reviewed by a cardiac radiologist with eight years of experience in the field of cardiovascular imaging who was blinded to the SPECT- 
MPI results. The myocardial features were reported as follows:

1- Muscle bridge location was defined as the "mid part" and "mid to distal part", according to its relation to arterial branches 1st diagonal artery (D1) and 2nd diagonal artery (D2) respectively.

2- Muscle bridge length was calculated as the distance between the point of entry into the myocardial layer and the point of exit from it.

3- Muscle bridge depth was calculated as the distance between the deepest point of the artery tunnel into the myocardial layer and the surface myocardial layer. MB is classified as regards to their depths: superficial $<2 \mathrm{~mm}$, borderline $\sim 2 \mathrm{~mm}$, and deep $>2 \mathrm{~mm}$.

4- LAD was divided into proximal, mid-part, and distal parts according to the diagonal arterial branches. The first part is a segment of LAD before the first diagonal branch, the mid-part is a segment of LAD between the first and second diagonal branches, and the distal part is a segment of LAD after the second diagonal branch. Furthermore, the mid to distal part is defined as a segment of the LAD at the pre to post second diagonal branch.

5- Proximal significant lesion was defined as greater than $50 \%$ reduction in diameter of the proximal part of the tunnel artery in the muscle bridge.

6- Diameter of LAD proximal to the muscle bridge (a): was defined as the diameter of the LAD artery before entering the muscle layer (myocardium) at the end of diastole in the short-axis, coronal and sagittal views.

7- Tunnel artery diameter in diastole (b): was defined as the diameter of the tunnel artery measured in the proxi$\mathrm{mal} 2 \mathrm{~mm}$ from the primary point of entry at the end of the diastole.

8- Tunnel artery diameter in systole (c) was defined as the diameter of the tunnel artery at the narrowest section.

9-Diameter difference was defined as the differences of the LAD diameter proximal to the muscle bridge and tunnel artery diameter in diastole $(\mathrm{a}-\mathrm{b})$.

10- Luminal narrowing: $(\mathrm{a}-\mathrm{c}) / \mathrm{a}$, the luminal diameter of MB was evaluated during the diastolic and systolic phases of the cardiac cycle to determine the effect of systolic compression upon the bridging segments to assume whether ischemia was induced by this luminal narrowing.

11- Systolic compression was defined as (b-c)/b.

12- Muscle index was defined as the multiplication of MB depth by MB length.

The interpretation of SPECT-MPI was done by a nuclear physician with twenty years of experience in this field of cardiac nuclear medicine. The physician was blinded to all CCTA results and a 17-segment model was used. The presence or absence of ischemia, segment involvement and the degree of decreased perfusion for each segment was qualitatively reported as mild, moderate, and severe.

\subsection{Statistical Analysis}

Numeric data were tested for normality using the Kolmogorov-Smirnov test. The two tailed independent samples t-test or Mann-Whitney U-test were used to compare the numeric data, like age and CCTA based parameters between the two groups. Fisher exact and Chi-square tests were used to evaluate nominal data distribution including sex, MB location, clinical sign, and intensity of ischemia between the two groups. Pearson's/Spearman's correlation tests were used to determine correlations between luminal narrowing and MB length (and depth) and between systolic compression index and MB length (and depth). Nominal and numeric data with non-normal distribution have been described as the median and percentage, respectively.

The area under the receiver operating characteristic (ROC) curve (AUC) was calculated for diameter difference, $\mathrm{MB}$ depth, and MB length in order to evaluate the overall performance of classification between the ischemia and non-ischemia groups. In this study, a P value of $<0.05$ was considered significant and AUC values were estimated beyond the 95\% confidence level. Statistical analysis was done using the SPSS software (IBM SPSS Statistics 19 for Windows, IBM Inc., Armonk, NY, USA).

\section{Results}

\subsection{Demographic Data of Patients}

This study included 32 patients (21 males and 11 females) with mean age of $48.19 \pm 11.70$ years. A total of 18 patients suffered from dyspnea while 22 patients had chest pain. Ten patients (31.25\%) had both signs of dyspnea and chest pain. MB was more prevalent in the men than in the women and this difference was statistically significant $(\mathrm{P}=$ 0.004; Table 1). MB was located in the mid part of LAD in 25 patients (78.1\%) and in the mid-to-distal part of LAD in seven patients. Superficial MB was observed in 15 patients, while deep MB and borderline cases occurred in 12 and five patients, respectively (Table 1 ).

\subsection{CCTA Finding}

The distribution of MB anatomic parameters in CCTA images is shown in Table 2. The length and depth of MB, diameter of LAD proximally to MB, tunnel artery diameter in diastole, and systole are $23.25 \pm 6.6,2.30 \pm 1.2,3.30 \pm 0.8$, $2.40 \pm 0.4$, and $1.70 \pm 0.5$, respectively. Also, evaluations of hemodynamic parameters of MB in patients are shown in Table 2. The mean of luminal narrowing, systolic compression, and systolic compression index are 40.3 $\pm 23.4,24 \pm$ 18 and $59.3 \pm 43$, respectively. 


\begin{tabular}{|c|c|c|c|c|}
\hline Variables & Total & Ischemic & Non-Ischemic & Pvalue \\
\hline Gender, No. (\%) & & & & 0.004 \\
\hline Female & $11(34.4)$ & $2(11.8)$ & $9(60)$ & \\
\hline Male & $21(65.6)$ & $15(88.2)$ & $6(40)$ & \\
\hline Frequency, No. & 15 & 5 & 12 & \\
\hline Percent, \% & 46.9 & 15.6 & 37.5 & \\
\hline
\end{tabular}

Abbreviation: MB, Myocardial Bridging.

Table 2. Distribution of Anatomic and Hemodynamic Myocardial Bridging Parameters in Coronary Computed Tomographic Angiography Images

\begin{tabular}{|c|c|c|c|c|c|}
\hline & \multicolumn{5}{|c|}{ Anatomic measurements } \\
\hline & Length & Depth & $\begin{array}{c}\text { Diameter of LAD proximal to } \\
\text { MB }\end{array}$ & $\begin{array}{c}\text { Tunnel artery diameter in } \\
\text { diastole }\end{array}$ & $\begin{array}{c}\text { Tunnel artery diameter in } \\
\text { systole }\end{array}$ \\
\hline Mean \pm SD & $23.25 \pm 6.6$ & $2.30 \pm 1.2$ & $3.30 \pm 0.8$ & $2.40 \pm 0.4$ & $1.70 \pm 0.5$ \\
\hline Minimum & 10 & 1.2 & 2 & 1.5 & 1.1 \\
\hline Maximum & 35 & 5 & 5 & 3.6 & 3.4 \\
\hline \multirow[t]{3}{*}{ Inter-quartile (25 - 75),\% } & $17-30$ & $1.5-3.5$ & $2.5-4.0$ & $2-2.6$ & $1.4-2.1$ \\
\hline & \multicolumn{5}{|c|}{ Hemodynamic measurements } \\
\hline & \multicolumn{2}{|c|}{ Luminal narrowing, \% } & \multicolumn{2}{|c|}{ Systolic compression, \% } & Muscle index, $\mathbf{m m}^{2}$ \\
\hline Mean \pm SD & \multicolumn{2}{|c|}{$40.3 \pm 23.4$} & \multicolumn{2}{|c|}{$24 \pm 18$} & $59.3 \pm 43$ \\
\hline Minimum & \multicolumn{2}{|c|}{0} & \multicolumn{2}{|c|}{0} & 15 \\
\hline Maximum & \multicolumn{2}{|c|}{75} & \multicolumn{2}{|c|}{50} & 150 \\
\hline Inter-quartile (25 - 75),\% & \multicolumn{2}{|c|}{$16.25-62.3$} & \multicolumn{2}{|c|}{$0.5-43$} & $30-103.7$ \\
\hline
\end{tabular}

Abbreviations: LAD, Left Anterior Descending; MB, Myocardial Bridging; SD, Standard Deviation.

\subsection{SPECT-MPI Finding}

Of the 32 patients, ischemia was observed in 17 patients (53.1\%, 15 males and two females), while 15 patients (46.9\%, six males and nine females) did not have signs of ischemia (Table 1). The median age of the ischemic and non-ischemic patients was 45 and 44 years and this difference was not significant $(\mathrm{P}=0.583)$. The locations of ischemia are described in Table 3 In these patients, the most frequent involvements were at the mid septal and anterior left ventricular (LV) walls, as well as the apico-septal and antero-apical walls of the heart. None of these patients presented severe ischemia. While most of the ischemia was mild and moderate, one patient with deep MB had fixed ischemia in the septal region (basal to apical) with the left bundle branch block (LBBB) pattern (Table 3).

\subsection{Compatibility of CCTA and SPECT-MPI Findings}

Evaluations were conducted for differences in dyspnea and chest pain in the ischemic and non-ischemic groups and these differences were determined as not statistically significant $(\mathrm{P}=0.755$ and $\mathrm{P}=0.599$, respectively) (Table 4). Also, there was a significant difference between the ischemic and non-ischemic groups for MB location and tunnel artery diameter in diastole $(\mathrm{P}=0.538$ and 0.166 , respectively) (Tables 4 and 5). There was a significant difference between the two groups for all other anatomic parameters and all hemodynamic parameters of MB (Table 5). Patients with superficial MB had no ischemia, while ischemia was observed in all patients with $\mathrm{MB}$ depth $\geq 2 \mathrm{~mm}$ (Figures 1 and 2). In the assessment of CCTA images in patients with MB with LAD arterial proximal segment, significant (> 50\% diameter reduction) narrowing is more among patients with positive ischemia as compared to the non-ischemic patients (percentiles 50 positive ischemia vs. non-ischemic patients: 62 vs. $15 ; \mathrm{P}=0.001$ ) (Table 5 ). The mean diameter difference after the entry of the artery to the muscle layer was $0.893 \pm 0.67 \mathrm{~mm}$. The difference in diameter was found to be significantly different between the two groups $(\mathrm{P}=0.001)$ (Table 5). 


\begin{tabular}{|c|c|c|c|c|c|c|}
\hline \multirow[t]{2}{*}{ Location } & \multirow[t]{2}{*}{ Frequency } & \multirow[t]{2}{*}{ Percent in ischemic group } & \multirow[t]{2}{*}{ Valid percent } & \multicolumn{3}{|c|}{ Intensity of ischemia } \\
\hline & & & & Mild-Moderate & Severe & Fixed \\
\hline Basal & 4 & 12.5 & 12.5 & 3 & 0 & 1 \\
\hline Mid-part & 11 & 64.7 & 34.4 & 10 & 0 & 1 \\
\hline Apical & 10 & 58.8 & 31.3 & 9 & 0 & 1 \\
\hline \multirow[t]{2}{*}{ Variables } & & & LAD territory ischemia & Total & \multirow{2}{*}{\multicolumn{2}{|c|}{ Pvalue }} \\
\hline & & No & Yes & & & \\
\hline \multicolumn{4}{|l|}{ Dyspnea } & & \multicolumn{2}{|c|}{0.755} \\
\hline \multicolumn{2}{|c|}{ No } & $7(46.7)$ & $7(41.2)$ & $14(43.8)$ & & \\
\hline \multicolumn{2}{|c|}{ Yes } & $8(53.3)$ & $10(58.8)$ & $18(56.3)$ & & \\
\hline \multicolumn{4}{|l|}{$\mathbf{C P}$} & & \multicolumn{2}{|c|}{0.599} \\
\hline \multicolumn{2}{|c|}{ No } & $4(26.7)$ & $6(35.3)$ & $10(31.3)$ & & \\
\hline \multicolumn{2}{|c|}{ Yes } & $11(73.3)$ & $11(64.7)$ & $22(68.8)$ & & \\
\hline \multicolumn{4}{|c|}{ MB location } & & \multicolumn{2}{|c|}{0.538} \\
\hline \multicolumn{2}{|c|}{ Mid Part } & $11(73.3)$ & $14(82.4)$ & $25(78.1)$ & & \\
\hline \multicolumn{2}{|c|}{ Mid-Distal Part } & $4(26.7)$ & $3(17.6)$ & $7(21.9)$ & & \\
\hline
\end{tabular}

Abbreviations: CP, Chest Pain; LAD, Left Anterior Descending; MB, Myocardial Bridging.

${ }^{\mathrm{a}}$ Values are expressed as No. (\%).

\begin{tabular}{|c|c|c|c|c|c|}
\hline \multirow[t]{2}{*}{ MB parameters } & \multicolumn{2}{|c|}{ Positive ischemia } & \multicolumn{2}{|c|}{ Negative ischemia } & \multirow[t]{2}{*}{ Pvalue } \\
\hline & Percentiles, 50 & Percentiles, 25-75 & Percentiles, 50 & Percentiles, 25-75 & \\
\hline Length, mm & 30 & $22.5-30$ & 20 & $15-25$ & 0.002 \\
\hline Depth, mm & 3.5 & $2-4$ & 1.5 & $1.2-1.5$ & 0.001 \\
\hline Diameter of LAD proximal to $\mathrm{MB}, \mathrm{mm}$ & 4 & $3.5-4.4$ & 2.5 & $2-3$ & 0.001 \\
\hline \multicolumn{6}{|l|}{ Tunnel artery diameter } \\
\hline Diastole & 2.5 & $2.2-2.8$ & 2.2 & $1.8-2.5$ & 0.166 \\
\hline Systole & 1.5 & $1.3-1.8$ & 2 & $1.6-2.5$ & 0.001 \\
\hline Luminal narrowing, \% & 62 & $51-66.3$ & 15 & $10-30$ & 0.001 \\
\hline Systolic compression, $\%$ & 43 & $25.5-48$ & 6 & $5-10$ & 0.001 \\
\hline Muscle index & 100 & $55-121$ & 30 & $18-35$ & 0.001 \\
\hline Diameter difference & 1 & $10-18$ & 0.4 & $2-5$ & 0.001 \\
\hline
\end{tabular}

Abbreviations: LAD, left Anterior Descending; MB, Myocardial Bridging.

\subsection{Ischemia Prediction Using CCTA Image}

Figures $3 \mathrm{~A}$ and $\mathrm{B}$ show a significant positive correlation between luminal narrowing, MB length and MB depth $(\mathrm{r}=$ $0.569, \mathrm{P}=0.001$ and $\mathrm{r}=0.789, \mathrm{P}=0.001$, respectively). In addition, systolic compression showed a significant positive correlation with MB length and MB depth $(r=0.568, P$ $=0.004$ and $0.840, \mathrm{P}=0.001$, respectively) (Figure $3 \mathrm{C}$ and
D).

Figure 4 and Table 2 show a ROC analysis of MB parameters in the diagnosis of ischemia. A diameter difference with a cut-off value of $0.65 \mathrm{~mm}$ can differentiate ischemic patients from non-ischemic patients with a sensitivity and specificity of 1 (95\% CI: 0.99 - 1.00) and 0.93 (95\% CI: 0.89 0.97), respectively and the area under the ROC curve (AUC) 

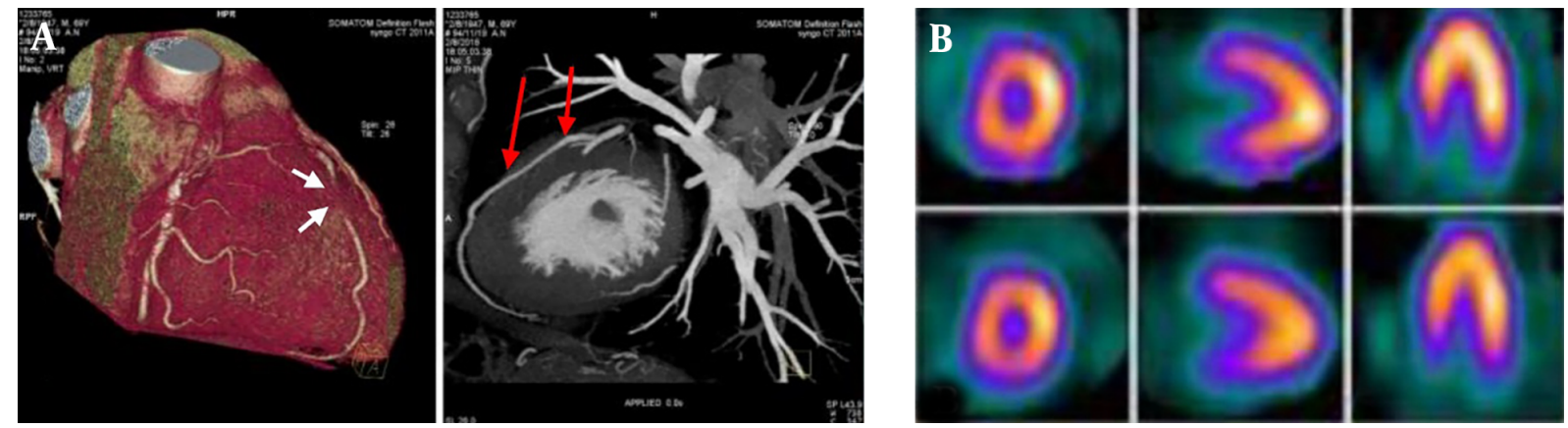

Figure 1. Volume-rendered technique (VRT) and maximum intensity projection (MIP) images show the muscle bridge of the left anterior descending (LAD) by 384 multi slice Siemens somatom force CT angiography (A) in a 70-year-old man with atypical chest pain. The myocardial bridging (MB) is located at the mid segment of LAD with a length of $35 \mathrm{~mm}$ and depth of $4 \mathrm{~mm}$ (two arrows indicate entrance and exit of MB). The selected slices of the gated SPECT images (B) shows mild to moderate ischemia in the mid anterolateral segment.
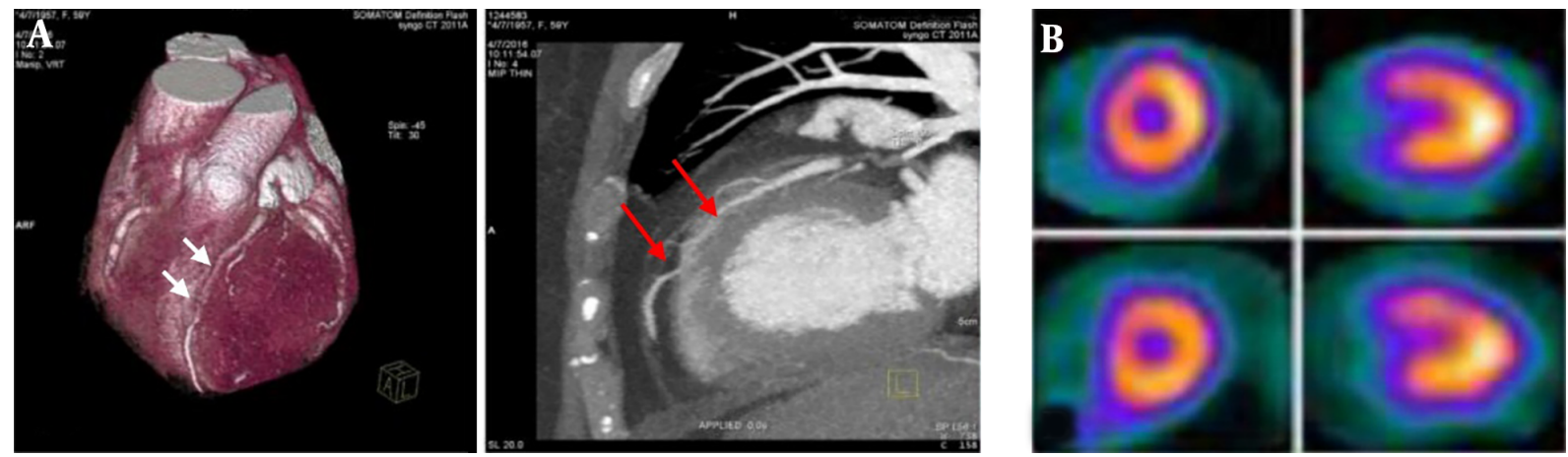

Figure 2. Volume-rendered technique(VRT) and maximum intensity projections (MIP) images show muscle bridge of left anterior descending (LAD) by 384 multi slice Siemen somatom force CT Angiography (A) in 59 years old female with atypical chest pain. The myocardial bridging (MB) is located at mid to distal segment of LAD in length of 20 $\mathrm{mm}$ and depth of $2.1 \mathrm{~mm}$ (two arrows indicate entrance and exit site of MB). Two selected short and long axis images of the gated SPECT (B) shows reversible perfusion defect in the mid anteroseptal segments.

was 0.996 (95\% CI: 0.983 - 1.000). Moreover, by considering MB depth cut-off value of $1.75 \mathrm{~mm}$, ischemia can be distinguished with a sensitivity and specificity of 1(95\% CI: 0.99 1.00), AUC of 1 (95\% CI: 0.99 - 1.00), MB length with $22.5 \mathrm{~mm}$ cut-off value had a lower discrimination power with 0.76 (95\% CI: 0.60 - 0.92) sensitivity, 67 (95\% CI: 0.51 - 0.83) specificity and AUC $=0.810$ (95\% CI: 0.661, 0.959) in the classification of patients into ischemic and non-ischemic groups (Table 6).

\section{Discussion}

MB has been considered as a benign condition, but recent studies have shown that it may lead to myocardial ischemia, infarction, coronary spasm, arrhythmias, and finally, death (17-19). Hence, detection of MB could be a critical factor for further reduction of complications and death. In the past decade, studies have shown that CCTA can depict MB with a higher rate than that reported by conventional angiography (19-22). In addition, conventional angiography is an invasive procedure and detecting MBs with significant hemodynamic effect on angiography remains cumbersome (23).

It seems that CCTA can characterize MB location, length, depth, definition of significant LAD arterial proximal segment ( $<50 \%$ reduction in diameter), narrowing assessment, proximal significant lesion, tunnel artery diameter in diastole and systole, diameter difference, and muscle index. These findings can be used to predict myocardial ischemia. MB depth and length were both shown to be promising predictive parameters for identifying patients with ischemia, as previously discussed. All MB with a depth greater than $1.75 \mathrm{~mm}$ had ischemia and MBs with a length greater than $22.5 \mathrm{~mm}$ also showed ischemia with an accuracy of $81 \%$ (Figure 4). Some efforts have been made to find the correlations between $\mathrm{MB}$ length and the presence of ischemia, but the findings remain controversial in 
A

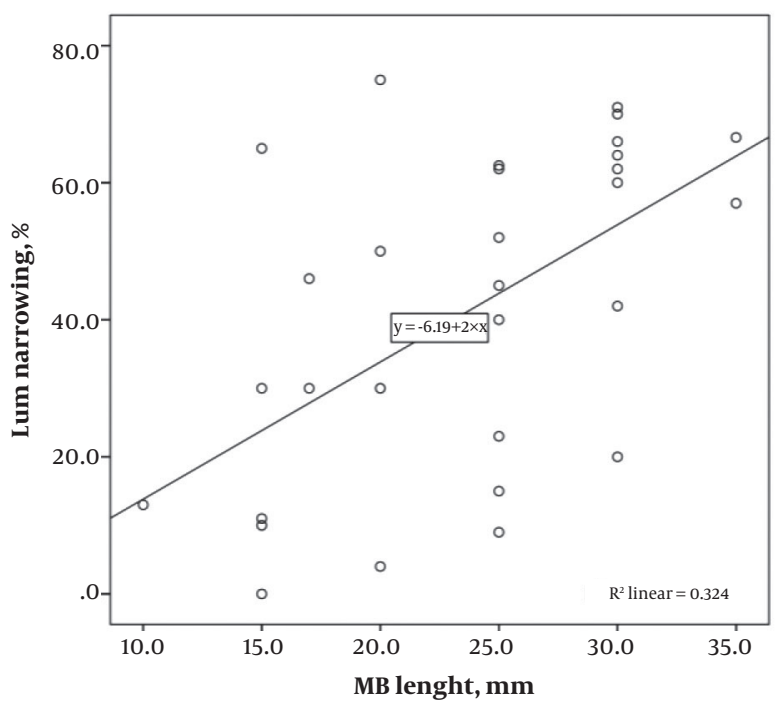

C

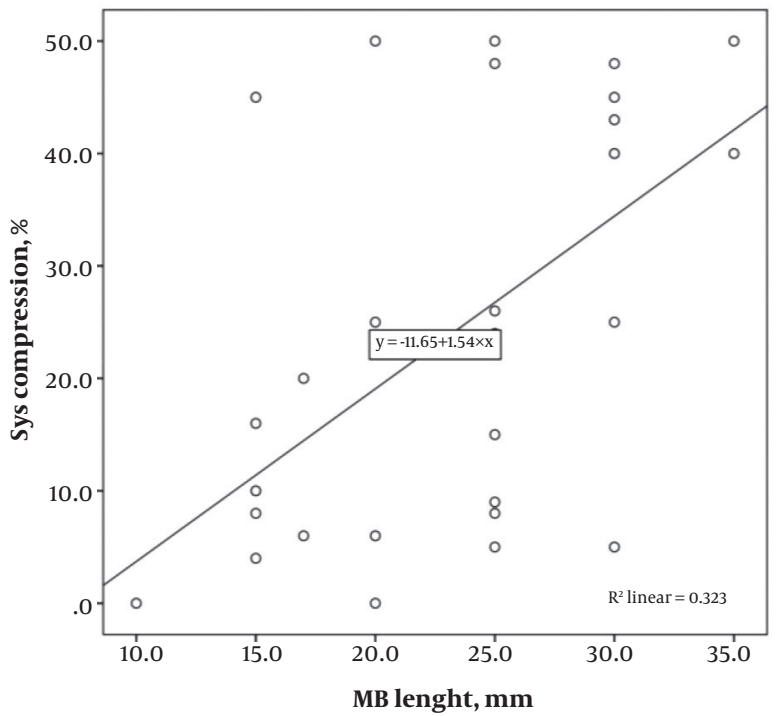

B

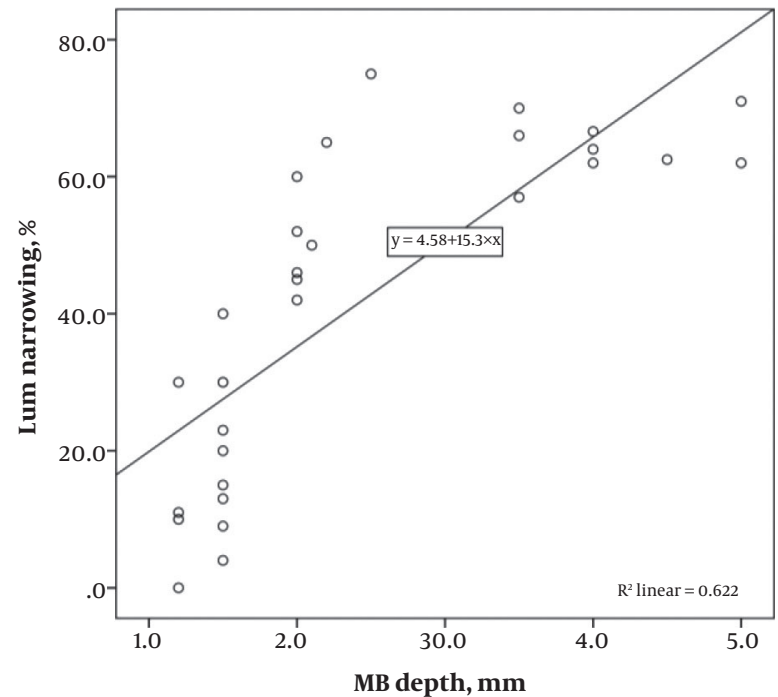

D

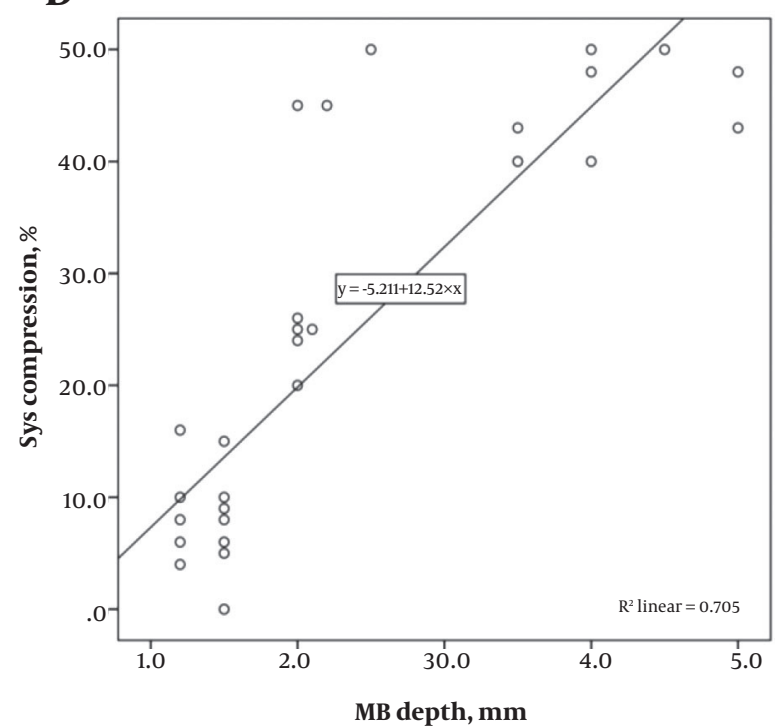

Figure 3. Scatter plot indicates there are significant positive correlations between: luminal narrowing and myocardial bridging $(\mathrm{MB})$ length with $\mathrm{R}^{2}=0.324$ (A); Narrowing and MB depth with $R^{2}=0.622(B)$; Systolic compression and MB length with $R^{2}=0.323(C)$; Systolic compression and $M B$ depth with $R^{2}=0.705$ (D).

\begin{tabular}{|c|c|c|c|c|}
\hline Variable & Cut-Off, mm & Sensitivity (95\% CI) & Specificity (95\% CI) & $\operatorname{AUC}(95 \% \mathrm{CI})$ \\
\hline MB depth & 1.75 & $1(0.99-1.00)$ & $1(0.99-1.00)$ & $1(0.991-1.000)$ \\
\hline Diameter difference & 0.65 & $1(0.99-1.00)$ & $0.93(0.89-0.97)$ & $0.996(0.983-1.000)$ \\
\hline MB length & 22.5 & $0.76(0.60-0.92)$ & $67(0.51-0.83)$ & $0.810(0.661-0.959)$ \\
\hline
\end{tabular}

Abbreviations: AUC, Area under ROC curve; MB, Myocardial Bridging.

the literature. While some studies showed that MB length correlated with ischemia and is in agreement with our re- 


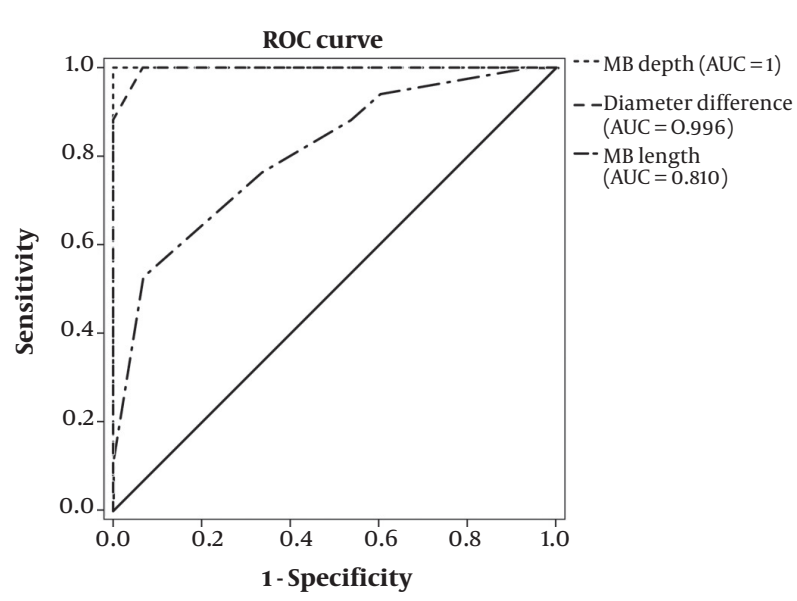

Figure 4. The receiver operating characteristic curves and area under the curves (AUCs) of myocardial bridging (MB) parameters

sults (24-26), other studies reported the opposite (27-29). The reason for the difference obtained in the results of this study compared to others could be related to the newer generation of force CT scan with high spatial and temporal resolution and the iterative reconstruction pattern for better demonstration of superficial from deep MB and its length at CCTA as compared to previous studies by Kim et al. (29) and Niu et al. (27) who used filter back projection reconstruction, as well as Wang et al. (28) who used conventional angiography instead of CCTA for demonstration of MB length as compared to SPECT-MPI. Furthermore, the population differences of patients could be another reason for the differences in MB length induced ischemia at SPECTMPI in previous studies as compared to the present study.

Furthermore, a diameter difference with a cut-off of $0.65 \mathrm{~mm}$ can diagnosis ischemia with $99.6 \%$ performance (Figure 4). Accurate and standardized measurement of these MB parameters is of great importance, since it can directly impact the patients' course of treatment. All patients with deep and long MB and history of ischemia could be considered for medical treatment in conjunction with surgical un-bridging or coronary artery bypass if nonrespondent to medical treatment (8). The results of the present study can be of help to physicians in predicting the risk of ischemia and thus, improve the treatment. Moreover, these results can also be of help to prevent unnecessary intervention, noncompulsory treatment, and avoidable complications.

In $\mathrm{MB}, \mathrm{LAD}$ diameters may reduce after entrance into the myocardia. Although recent studies have mentioned this phenomenon $(26,30)$, to the best of our knowledge, this is the first study to evaluate the effect of this phenomenon on the prediction of ischemia.
There has been increasing incidence of detection of $\mathrm{MB}$ with the advent of the second and recently the third generation of multi-slice CT scanner (31). In this study, Dualsource 384 slice-CT was used to assess MB morphology and the results showed that MB parameters could predict ischemia with high accuracy (Table 6).

Since parameters such as age and clinical signs were not significantly different between the ischemic and nonischemic patients, these parameters would not act as confounding factors in our study. Also, none of the patients has any underlying disease such as valve disease, atherosclerosis and myocardial hypertrophy that could ultimately affect the results.

There were some limitations in our study. First, the number of patients was small, further investigation using a larger data set would be appropriate. Second, patients were not followed up for a long term and there was no clear picture of how this ischemia-induced test would ultimately result in prognosis.

In conclusion, the results of this study showed that CCTA could help physicians to detect ischemia in MB candidates. Further study may be needed to evaluate the impact of muscle index and luminal narrowing in the prediction of significant hemodynamic MBs.

\section{Footnotes}

Authors' Contributions: None declared.

Conflicts of Interest: None.

Ethical Approval: This study was approved by the ethics committee of Iran University of Medical Sciences (No. IR.IUMS.FMD.REC 1396.9211171026).

Financial Disclosure: None declared.

Funding/Support: This work was supported by a grant (No. 9211171026) from Iran University of Medical Sciences (IUMS).

\section{References}

1. Rovai D, Di Bella G, Pingitore A, Coceani M. Myocardial bridging: a review with emphasis on electrocardiographic findings. Ann Noninvasive Electrocardiol.2015;20(2):103-7. doi:10.1111/anec.12242. [PubMed: 25530097].

2. Hazirolan T, Canyigit M, Karcaaltincaba M, Dagoglu MG, Akata D, Aytemir K, et al. Myocardial bridging on MDCT. AJR Am J Roentgenol. 2007;188(4):1074-80. doi:10.2214/AJR.06.0417. [PubMed: 17377050].

3. Alegria JR, Herrmann J, Holmes DJ, Lerman A, Rihal CS. Myocardial bridging. Eur Heart J. 2005;26(12):1159-68. doi: 10.1093/eurheartj/ehi203. [PubMed: 15764618].

4. Angelini P, Trivellato M, Donis J, Leachman RD. Myocardial bridges: a review. Prog Cardiovasc Dis. 1983;26(1):75-88. doi: 10.1016/00330620(83)90019-1. [PubMed: 6346395]. 
5. Kim PJ, Hur G, Kim SY, Namgung J, Hong SW, Kim YH, et al. Frequency of myocardial bridges and dynamic compression of epicardial coronary arteries: a comparison between computed tomography and invasive coronary angiography. Circulation. 2009;119(10):1408-16. doi: 10.1161/CIRCULATIONAHA.108.788901. [PubMed: 19255347].

6. Porstmann W, Iwig J. [Intramural coronary vessels in the angiogram]. Fortschr Geb Rontgenstr Nuklearmed. 1960;92:129-33. doi: 10.1055/s0029-1226492. [PubMed:14434245].

7. Konen E, Goitein O, Sternik L, Eshet Y, Shemesh J, Di Segni E. The prevalence and anatomical patterns of intramuscular coronary arteries: a coronary computed tomography angiographic study. J Am Coll Cardiol. 2007;49(5):587-93. doi: 10.1016/j.jacc.2006.09.039. [PubMed: 17276183].

8. Ge J, Jeremias A, Rupp A, Abels M, Baumgart D, Liu F, et al. New signs characteristic of myocardial bridging demonstrated by intracoronary ultrasound and Doppler. Eur Heart J. 1999;20(23):1707-16. doi: 10.1053/euhj.1999.1661. [PubMed: 10562478].

9. Schwarz ER, Klues HG, vom Dahl J, Klein I, Krebs W, Hanrath P. Functional characteristics of myocardial bridging. A combined angiographic and intracoronary Doppler flow study. Eur Heart J. 1997;18(3):434-42. doi: 10.1093/oxfordjournals.eurheartj.a015263. [PubMed: 9076380].

10. Kantarci M, Duran C, Durur I, Alper F, Onbas O, Gulbaran M, et al. Detection of myocardial bridging with ECG-gated MDCT and multiplanar reconstruction. AJR Am J Roentgenol. 2006;186(6 Suppl 2):S391-4 doi: 10.2214/AJR.05.0307. [PubMed: 16714614].

11. Thomson V, Botnar R, Croisille P. Usefulness of MRI to demonstrate the mechanisms of myocardial ischemia in hypertrophic cardiomyopathy with myocardial bridge. Cardiology. 2007;107(3):159-64. doi: 10.1159/000094746. [PubMed: 16888385].

12. Javadrashid R, Tarzamni MK, Aslanabadi N, Ghaffari M, Salehi A, Sorteji $\mathrm{K}$. Myocardial bridging and coronary artery anomalies detected by ECG-gated 64-row multidetector computed tomography angiography in symptomatic patients. Folia Morphol (Warsz). 2009;68(4):2016. [PubMed: 19950067].

13. Stănescu A, Suciu Z, Rat N, Morariu M, Chitu M. CT-based assessment of myocardial bridging in patients with acute chest pain and no atherosclerotic etiology. J Cardiovasc Emerg. 2016;2(2). doi: 10.1515/jce2016-0011.

14. Motarjem P, Zimmerman S. Pearls and Pitfalls in Cardiovascular Imaging: Pseudolesions, Artifacts and Other Difficult Diagnoses. Cambridge University Press; 2015.

15. Hesse B, Tagil K, Cuocolo A, Anagnostopoulos C, Bardies M, Bax J, et al. EANM/ESC procedural guidelines for myocardial perfusion imaging in nuclear cardiology. Eur J Nucl Med Mol Imaging. 2005;32(7):855-97. doi: 10.1007/s00259-005-1779-y. [PubMed: 15909197].

16. Sabharwal NK, Lahiri A. Role of myocardial perfusion imaging for risk stratification in suspected or known coronary artery disease. Heart. 2003;89(11):1291-7. doi: 10.1136/heart.89.11.1291. [PubMed: 14594881]. [PubMed Central: PMC1767933].

17. Vales L, Kanei Y, Fox J. Coronary artery occlusion and myocardial infarction caused by vasospasm within a myocardial bridge. J Invasive Cardiol. 2010;22(4):E67-9. [PubMed: 20351399].

18. Li JJ. Is myocardial bridging a bridge connecting to cardiovascular events? Chin Med J (Engl). 2010;123(7):964-8. [PubMed: 20497696].
19. Li ZF, Yang SG, Ge JB. Acute myocardial infarction due to myocardial bridge. Chin Med J (Engl). 2012;125(19):3589-90. [PubMed: 23044332].

20. Nieman K, Coenen A. Myocardial Enhancement Defects on CT Angiograms: Promising Prognosticator or Water Under the Bridge? JACC Cardiovasc Imaging. 2015;8(6):695-6. doi: 10.1016/j.jcmg.2015.02.011. [PubMed: 26068286].

21. La Grutta L, Runza G, Lo Re G, Galia M, Alaimo V, Grassedonio E, et al. Prevalence of myocardial bridging and correlation with coronary atherosclerosis studied with 64-slice CT coronary angiography. Radiol Med. 2009;114(7):1024-36. doi: 10.1007/s11547-009-0446-y. [PubMed: 19697102].

22. Kim SY, Lee YS, Lee JB, Ryu JK, Choi JY, Chang SG, et al. Evaluation of myocardial bridge with multidetector computed tomography. Circ J. 2010;74(1):137-41. doi: 10.1253/circj.CJ-09-0407. [PubMed: 19966506].

23. Dominguez B, Valderrama V, Arrocha R, Lombana B. [Myocardial bridging as a cause of coronary insufficiency]. Rev Med Panama. 1992;17(1):28-35. [PubMed: 1603985].

24. Nakanishi R, Rajani R, Ishikawa Y, Ishii T, Berman DS. Myocardial bridging on coronary CTA: an innocent bystander or a culprit in myocardial infarction? J Cardiovasc Comput Tomogr. 2012;6(1):3-13. doi: 10.1016/j.jcct.2011.10.015. [PubMed: 22264630].

25. De Giorgio F, Grassi VM, Polacco M, Pascali VL, d'Aloja E, Arena V. Myocardial bridging and sudden cardiac death: is the actual classification exhaustive? Int J Cardiol. 2014;172(3):e383-4. doi: 10.1016/j.ijcard.2013.12.286. [PubMed: 24508108].

26. Arjmand Shabestari A, Azma R, Nourmohammad A, Shakiba M. Systolic Compression of a Myocardial Bridged Coronary Artery and its Morphologic Characteristics: A Combination Study of Computed Tomography Angiography and Invasive Angiography. Iran J Radiol. 2016;13(4). e31647. doi: 10.5812/iranjradiol.31647. [PubMed: 27895870]. [PubMed Central: PMC5116748].

27. Niu YJ, Zhang XL, Cao AD, Leng B. Clinical value of the correlations of mural coronary artery compression extent with myocardial bridge length and thickness evaluated by 128-slice CT. Exp Ther Med. 2013;5(3):848-52. doi: 10.3892/etm.2012.879. [PubMed: 23408740]. [PubMed Central: PMC3570202].

28. Wang D, Sun JP, Lee AP, Ma GS, Yang XS, Yu CM, et al. Evaluation of left ventricular function by three-dimensional speckle-tracking echocardiography in patients with myocardial bridging of the left anterior descending coronary artery. J Am Soc Echocardiogr. 2015;28(6):674-82. doi: 10.1016/j.echo.2015.02.012. [PubMed: 25795472].

29. Kim SS, Ko SM, Song MG, Hwang HG. Systolic luminal narrowing and morphologic characteristics of myocardial bridging of the midleft anterior descending coronary artery by dual-source computed tomography. Int J Cardiovasc Imaging. 2011;27 Suppl 1:73-83. doi: 10.1007/s10554-011-9959-2. [PubMed: 21997576].

30. Aydin A, Cubuk R, Atasoy MM, Gurol T, Soylu O, Dagdeviren B. The morphologic and functional features of LAD myocardial bridging at multi-detector computed tomography coronary angiography: correlation with coronary artery disease. Turk Kardiyol Dern Ars. 2015;43(1):31-7. doi: 10.5543/tkda.2015.23672. [PubMed: 25655848].

31. Görmeli CA, Yağmur, J, Özdemir, R, Özdemir, Z. M, Sağır, A, Kahraman, C. C . Comparison of myocardial bridging prevalence using 64-slice versus 256-slice computed tomography scanners: What has changed with recent innovations in CT? Biomed Res. 2016;27(3):954-8. 